\title{
Synthetic Genes for Artificial Ants. Diversity in Ant Colony Optimization Algorithms
}

\author{
S.C. Negulescu, I. Dzitac, A.E. Lascu
}

\author{
Sorin C. Negulescu \\ "Lucian Blaga" University of Sibiu, "Hermann Oberth" Faculty of Engineering \\ 10, Victoriei Bd, 550024 Sibiu, Romania \\ Email: sorin.negulescu@ulbsibiu.ro \\ Ioan Dzitac \\ "Aurel Vlaicu" University of Arad, Faculty of Exact Sciences, \\ Department of Mathematics-Informatics, \\ Str. Elena Dragoi, Nr. 2, Complex Universitar M, Arad, Romania \\ and \\ R\&D Agora Ltd. Oradea [Cercetare Dezvoltare Agora] \\ 8, Piata Tineretului, 410526 Oradea, Romania \\ E-mail ioan.dzitac@uav.ro \\ Alina E. Lascu \\ "Lucian Blaga" University of Sibiu, "Hermann Oberth" Faculty of Engineering \\ 10, Victoriei Bd, 550024 Sibiu, Romania \\ E-mail alina.lascu@ulbsibiu.ro
}

\begin{abstract}
Inspired from the fact that the real world ants from within a colony are not clones (although they may look alike, they are different from one another), in this paper, the authors are presenting an adapted ant colony optimisation (ACO) algorithm that incorporates methods and ideas from genetic algorithms (GA). Following the first (introductory) section of the paper is presented the history and the state of the art, beginning with the stigmergy and genetic concepts and ending with the latest ACO algorithm variants as multiagent systems (MAS). The rationale and the approach sections are aiming at presenting the problems with current stigmergy-based algorithms and at proposing a (possible - yet to be fully verified) solution to some of the problems ("synthetic genes" for artificial ants). A model used for validating the proposed solution is presented in the next section together with some preliminary simulation results. Some of the conclusions regarding the main subject of the paper (synthetic genes: agents within the MAS with different behaviours) that are closing the paper are: a) the convergence speed of the ACO algorithms can be improved using this approach; b) these "synthetic genes" can be easily implemented (as local variables or properties of the agents); c) the MAS is self-adapting to the specific problem that needs to be optimized.

Keywords: Ant Colony Optimization, Genetic Algorithms, Multiagent Systems, Stigmergy.
\end{abstract}

\section{Introduction}

There are about ten thousand species of ants living on earth, grouped in colonies, all of them having or one or (at most) two queens and sterile female workers (all the other ants). As the research conducted by biologists showed [5], there is no central control or no management whatsoever inside a colony, but the behaviour of the colony changes (as it gets older and larger). It was also determined that there are four types of ants [5]: harvester ants, foraging ants (out on a foraging trail searching for food or bringing food back), patrolling (out early in the morning before the foragers are active, somehow choosing the directions that the forager ants will go, and just by coming back to the nest they indicate to the foragers that is safe to go out), maintenance (work inside the nest with moist soil for building the walls), midden work (put some kind of territorial chemical in the garbage they create). Another interesting fact is that $50 \%$ of the ants are doing nothing, acting as a reserve for exceptionally situations.

One class of multi-agent systems (MAS) are copying some of the characteristics of the foraging ants within the colonies (depending on how much these characteristics are helpful in solving a particular type of problem) such as [10]: 
- being a distributed society of autonomous individuals/agents;

- having fully distributed control among the agents;

- having localized communications among the individual;

- taking stochastic decisions;

- having system-level behaviours that are transcending the behavioural repertoire of the single (minimalist) agent;

- following simple interaction rules.

As a result, the overall very important features of these systems are: robustness, adaptability and scalability.

The rest of this paper is arranged as follows: the history section presents the stigmergy and genetic concepts and variants of ACO algorithms as MAS. The rationale and the approach sections are aiming at presenting the problems with current stigmergy-based algorithms and at proposing a (possible - yet to be fully verified) solution to some of the problems ("synthetic genes" for artificial ants). A model used for validating the proposed solution is presented in the next section together with some preliminary simulation results. Some of the conclusions regarding the main subject of the paper presented in the last section are: a) the convergence speed of the ACO algorithms can be improved using this approach; b) these "synthetic genes" can be easily implemented (as local variables or properties of the agents); c) the MAS is self-adapting to the specific problem that needs to be optimized.

\section{History and related work}

Swarm intelligence systems (inspired from biological systems as ant colonies, bird flocking, animal herding, bacterial growth, and fish schooling) are in fact simple agents that are interrelated, being able to communicate one with another and to interact with their environment. They follow simple rules and there is no centralized control [9]. In swarm intelligence systems the focus is not on modelling, inspired from biologic (sub-symbolic) paradigms, but merely on simulating biologic behaviour [8].

The ant colony optimization algorithm is a probabilistic technique for solving computational problems which can be reduced to finding good paths through graphs [17]. This technique was initially proposed by Marco Dorigo in 1992 in his $\mathrm{PhD}$ thesis [6] [7] and it was aiming at searching an optimal path in a graph (i.e. the travelling salesperson problem - TSP) based on the behaviour of ants seeking a path between their colony and a source of food [4].

The algorithm was further developed and, as a result, some common extensions appeared such as the Elitist Ant System (EAS) where the ant that found a better solution could deposit an extra amount of pheromone; the Max-Min Ant System (MMAS)[16] where the pheromone intensity was bounded to certain minimum and maximum values (Tmax, Tmin), and where only the ant that found a better solution could deposit pheromone. The MMAS was the first algorithm that was constantly checking its convergence to solution; all the edges in the graph were initialized to Tmax and reinitialized to Tmax when the algorithm was near stagnation [17].

Another version of the ACO is the Rank-Based Ant System (ASrank) where all solutions are ranked according to their fitness. The amount of pheromone deposited is then weighted for each solution, such that the solutions with better fitness deposit more pheromone than the solutions with worse fitness [17].

Besides these common variants of the algorithm [12], the authors proposed some different approaches such as:

- Human-Driven Stigmergic Control [3] - aimed at exploring the relationship between stigmergy and synergy, based on the threshold principle, specifically, focusing on affordability and keeping a definite engineering perspective, the purpose was to save computer resources in applying stigmergic control to industrial problems by exploring the relationship between the number of digital ants and problem complexity. The long-range target was to follow the analogy to superconductivity: moving the threshold in order to improve performance and/or save computing resources. The research proved that the threshold exists and it depends on problem type and complexity; the same solution quality can be obtained with fewer ants.

- User-Driven Heuristics [1] [12] - the approach emphasised the significance of the environment in the agent system and the attention that must be paid to the dynamics that emerges from the indirect interactions of the agents. Finding out the form and parameters that influence the system behaviour is non-trivial for humans and the research investigated the methods that would enable user-driven solutions in dealing with system tuning. The proposed methods were based on two essential functional requirements: a) at the macro level, the need to monitor and represent in an intuitive way the system behaviour and b) at the micro level, the need to control and track the system state space. The approach proved to be workable on usual configurations and effective in dealing with combinatorial explosion. 
- (Sub-)Symbolic Inferences in Multi-Agent Systems [2] - the research aimed at showing that new logics, already useful in modern software engineering, become necessary mainly for MAS, despite obvious adversities. The features asked for by the paradigm of computing as intelligent interaction, based on "nuances of nuancedreasoning", that should be reflected by agent logics were outlined. By injecting symbolic reasoning in systems based on sub-symbolic "emergent synthesis" the authors presented the way in which how quantifiable synergy can be reached - even in advanced challenging domains, such as stigmergic coordination.

The original idea has diversified to solve a wider class of numerical problems, and as a result, several problems have emerged, drawing on various aspects of the behaviour of ants such as solving combinatorial optimization, classification and image processing problems [15].

The authors successfully applied the ACO algorithms to solve difficult problems such as: vehicle route allocation (with multiple constraints) [13] [14] and optimal capacitor banks placement in power distribution networks [15] where the criterion of the mathematical optimization model was a nonlinear function based on costs and the model imposed equality constraints described by the network operating equations and inequality constraints required to maintain within admissible limits the parameters characterizing the system state.

\section{Rationale and approach}

The motive for proposing synthetic genes for artificial ants and promoting the diversity in ant colony optimization algorithms is that the convergence speed of current variations of ACO algorithms is strongly dependent of the problem type and size. The real world ants from within a colony are not clones as current ACO algorithms are modelling them; although they may look alike, they are different from one another.

The vast majority of ACO algorithms have general variables that are determining the overall system behaviour such as:

- pheromone evaporation speed when updating the pheromones intensities in the graph $(\rho)$;

- pheromone quantity deposited on graph (Q);

- pheromone influence is on edge selection $(\alpha)$;

- distance influence on edge selection $(\beta)$;

- the multiplication value for the pheromone intensity of the elitist ant $(\varepsilon)$ - only for EAS.

When a certain problem must be solved, the values of the above presented parameters are set and after a few runs they are adjusted in order to improve the overall convergence speed or solution quality.

When dealing with dynamic optimisation problems the above mentioned approach accentuates once more its weak points, as it is almost impossible to determine good parameter values.

A possible manner to combat these limitations is to adopt some ideas from the GA field. Although hybrid approaches between MAS (such as ACO or EAS) and GA are not new, the technique proposed herein is innovative because:

- it does not absolutely require to implement all the GA concepts (like selection, crossover and mutation);

- the fitness function may or may not exist (depending on problem type);

- the generations from GA can be associated to the iteration number from MAS;

- the global parameters belonging to the algorithm are converted to local parameters belonging to ants.

As depicted in figure 1b, each virtual ant within the colony has its own governing parameters in the same manner that real ants exhibit different behaviour. More details about the way in which such a system should work are presented in the next section.

\section{The model}

Since the model proposed herein imposes that each virtual ant within the colony must have its own parameters that are determining its behaviour, it is important for the user to have the ability to specify some general limits in which these parameters may vary. In this regard, some other general parameters must be defined as follows:

- minimum and maximum values for the exploitation of the good old found paths - $\alpha_{\min }, \alpha_{\max }$;

- minimum and maximum values for the exploration of the other paths within the graph - $\beta_{\min }, \beta_{\max }$;

- minimum and maximum values for the multiplication value for the pheromone intensity of the (elitist) ants that found a better solution - $\varepsilon_{\max }, \varepsilon_{\max }$; 


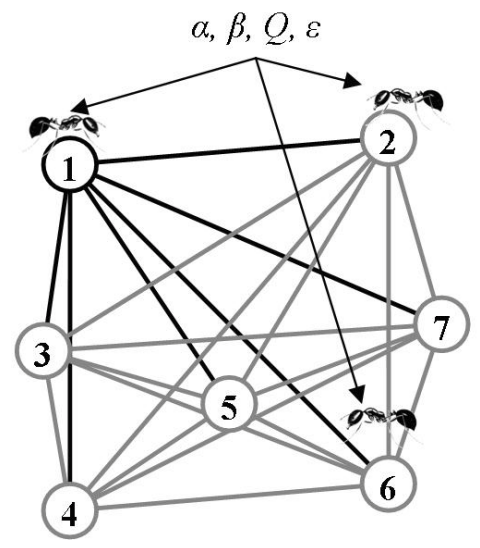

(a)

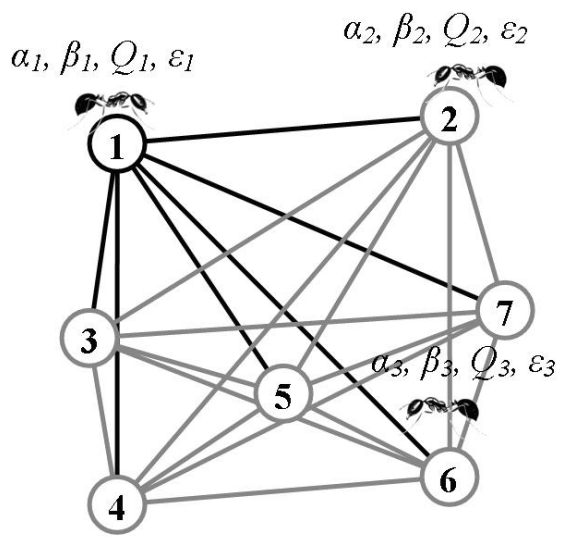

(b)

Figure 1: Exemplification of a) global parameters versus b) local parameters for the ant system

Depending on the problem to be solved some other global parameters can be defined to enforce the limits for the local parameters (e.g. for the vehicle route allocation problem [13] a local history parameter was used and $H_{\text {min }}, H_{\text {max }}$ as global parameters).

After finding a better solution, different actions can be taken by the algorithm to auto-tune the ant local parameters:

- the parameters will be adjusted for some, all or none of the ants;

- parameters can be adjusted using different types of equations - e.g. form simple average, weighted average (depending on solution quality), or other linear functions to polynomial or exponential functions.

The initialization of the ant parameters can be easily done in a random manner as shown in figure 2, but some other initialization methods can be applied as well (e.g. Gaussian centred to the average value: $\min +(\max -\min ) / 2$ ) .

Although the research on the proposed model is in progress the results so far are promising. The algorithm is self-tuning to the problem type and size. Some other methods must be checked such as starting with a big number of ants and reducing it to a minimum in time, with a twofold advantage:

- having a large genetic pool at the beginning will assure that the algorithm can adapt to very diverse problems;

- reducing the number of ants will increase the computation speed; this number can be drastically reduced as presented in [3]. 


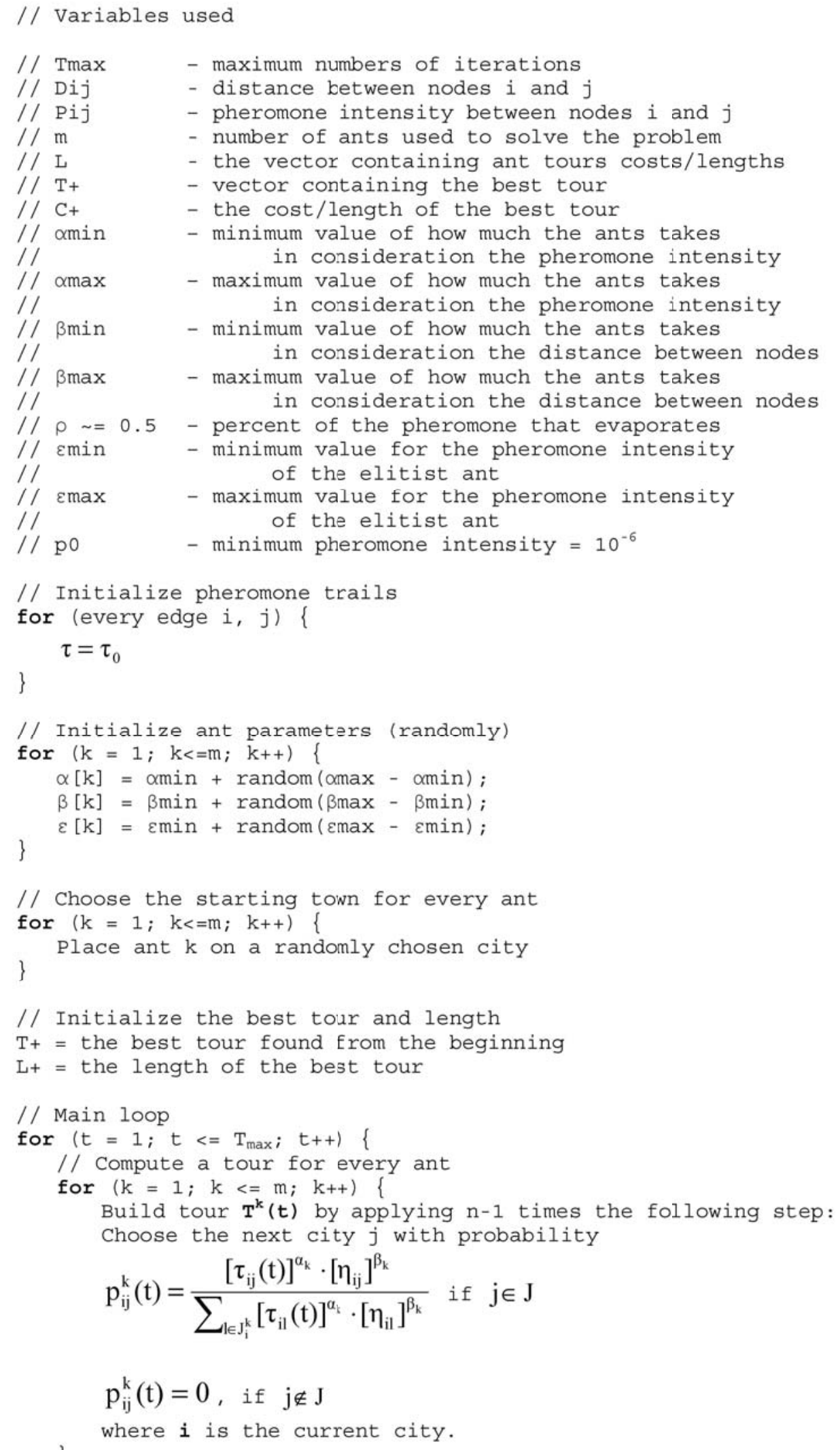




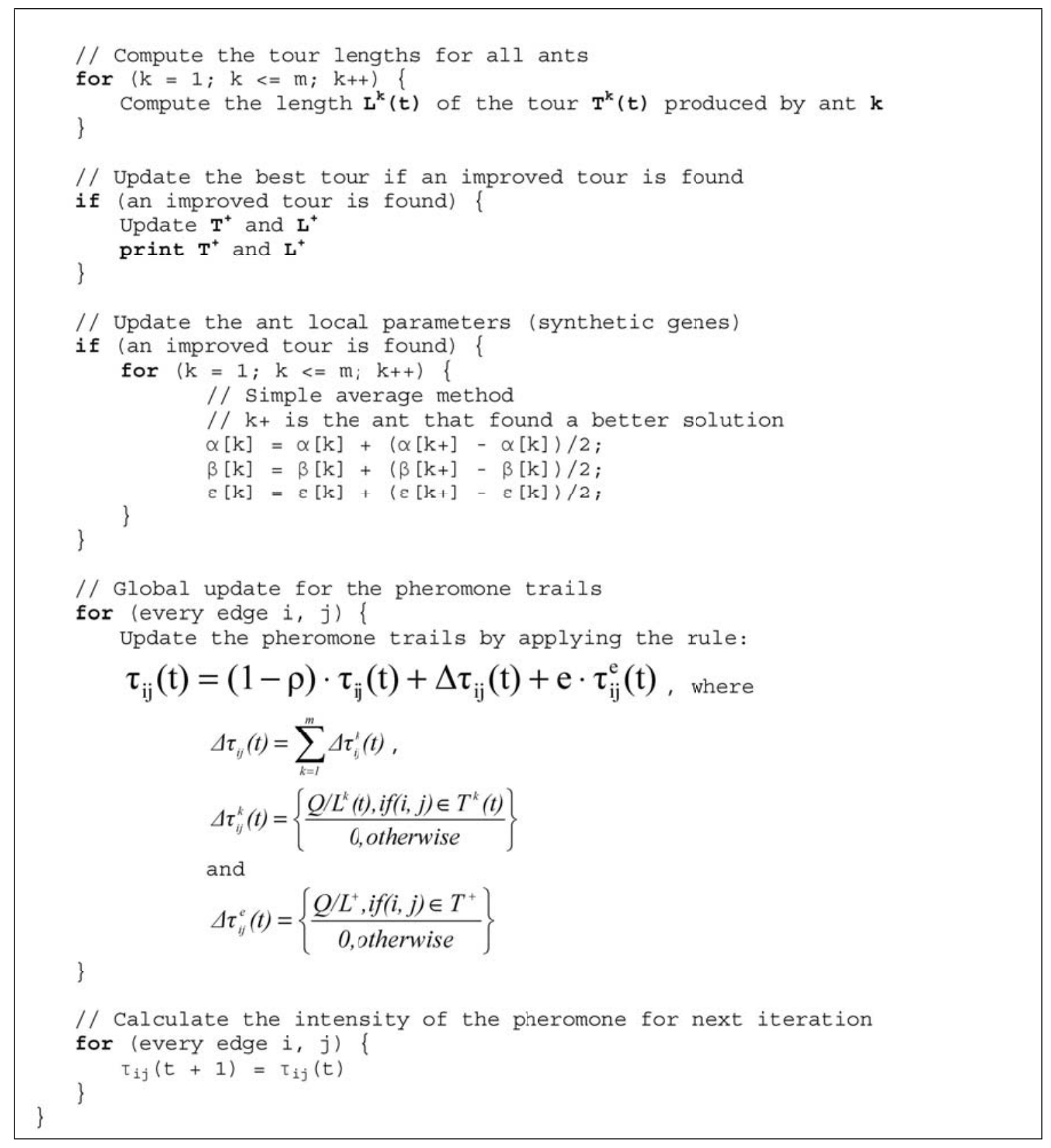

Figure 2: The proposed pseudocode for the "synthetic genes" enhanced EAS. 


\section{Conclusions and future work}

Since this paper presents an ongoing research, the conclusions will be presented tanking in consideration two time ranges: a) short (current relevant results) and b) medium-long (directions of future work).

a1. The proposed ACO algorithms have the ability to self-adapt to different optimisation problem types and sizes.

a2. The convergence speed of the ACO algorithms can be improved using this approach.

a3. The "synthetic genes" can be easily implemented (as local variables or properties of the agents); only some other global variables that will impose the limits of variation must be defined.

a4. Different types of initialisation of the genes values can be used (e.g. random). Ideas for this particular subject can be used from the GA field.

a5. The genes values can be automatically adjusted for some, all or none of the ants. Moreover, this can be achieved using different types of equations - e.g. form simple average, weighted average (depending on solution quality), or other linear functions to polynomial or exponential functions.

b1. Some other methods of initialization/algorithm evolution must be tested. As presented in the previous section, the algorithm can start with a big number of ants and reduce it to a minimum in time.

b2. Other concepts form GA can be implemented taking into account both the advantages (solution quality) and the disadvantages (computing time) like selection, crossover or mutation.

b3. Finding a solution to the stagnation problem that is intrinsic both to ACO and GA approaches. Another possible technique would be to reinitialize the local parameters while keeping the pheromones intensities on the graph intact.

\section{Bibliography}

[1] Bărbat B.E, Moiceanu A., Pleşca S., Negulescu S.C., Affordability and Paradigms in Agent-Based Systems, Computer Sc. J. of Moldova, 15, 2(44):178-201, 2007.

[2] Bărbat B.E., Negulescu S.C., From Algorithms to (Sub-)Symbolic Inferences in Multi-Agent Systems. International Journal of Computers, Communications and Control, 1(3):5-12, 2006.

[3] Bărbat B.E., Negulescu S.C., Zamfirescu C.B., Human-Driven Stigmergic Control. Moving the Threshold. In N. Simonov (Ed.), Proc. of the 17th IMACS World Congress (Scientific Computation, Applied Mathematics and Simulation), Paris, pp.86-92, 2005.

[4] Bonabeau E., Dorigo M., Theraulaz G., Swarm Intelligence: From Natural to Artificial Systems, New York: Oxford University Press, 1999.

[5] Deborah Gordon (2008), Dig ants, Retrived 11.2009, from TED. Ideas worth spreading. Web site: http://www.ted.com/talks/lang/eng/deborah_gordon_digs_ants.html.

[6] Dorigo M., Maniezzo V., Colorni A., The Ant System: Optimisation by a Colony of Cooperating Agents, IEEE Transactions on Systems, Man, and Cybernetics, Part B, 26 (1):29-42, 1996.

[7] Dorigo, M., Optimization, Learning and Natural Algorithms, PhD thesis. Italy: Politecnico di Milano, 1992.

[8] Dzitac I., Bărbat B.E., Artificial Intelligence + Distributed Systems = Agents, International Journal of Computers, Communications and Control, 4(1):17-26, 2009.

[9] Dzitac I., Moisil I., Advanced AI Techniques for Web Mining, Proceedings of the 10th WSEAS international conference on Mathematical methods, computational techniques and intelligent systems, Corfu, pp.343-346, 2008.

[10] Gambardella L.M., Di Caro G. (2005), The Ant Colony Optimization (ACO) Metaheuristic: a Swarm Intelligence Framework for Complex Optimization Tasks. Retrived 2008, from University of Bologna: First Summer School on Aspects of Complexity. Web site: http://www.cs.unibo.it/ fioretti/AC/AC2005/ docs/slides_dicaro.pdf.

[11] Negulescu S.C., Bărbat B.E., Enhancing the effectiveness of simple multi-agent systems through stigmergic coordination, In ICSC-NAISO (Ed.), Fourth International ICSC Symposium on Engineering Of Intelligent Systems (EIS 2004), Canada: ICSC-NAISO Academic Press, pp.149-156, 2004. 
[12] Negulescu S.C., Zamfirescu C.B., Bărbat B.E., User-Driven Heuristics for nondeterministic problems. Studies in Informatics and Control (Special issue dedicated to the 2nd Romanian-Hungarian Joint Symp. on Applied Computational Intelligence), 15(3):289-296, 2006.

[13] Negulescu, S.C., Kifor, C.V., Oprean, C., Ant Colony Solving Multiple Constraints Problem: Vehicle Route Allocation. International Journal of Computers, Communications and Control (IJCCC), 3(4):366-373, 2008.

[14] Negulescu, S.C., Oprean, C., Kifor, C.V., Carabulea, I., Elitist ant system for route allocation problem. In Mastorakis, N.E. et al (Ed.), Proceedings of the 8th conference on Applied Informatics and Communications, Greece: World Scientific and Engineering Academy and Society (WSEAS), pp.62-67, 2008.

[15] Secui, D.C., Dzitac, S., Bendea, G.V., Dzitac, I., An ACO Algorithm for Optimal Capacitor Banks Placement in Power Distribution Networks. Studies in Informatics and Control, 18(4):305-314, 2009.

[16] Stützle T., Hoos H.H., MAX-MIN Ant System, Future Generation Computer Systems, 16 (8):889-914, 2000.

[17] Wikipedia, the free encyclopedia (2009), Ant colony optimization. Retrived 11.2009, from WIkipedia. Web site: http://en.wikipedia.org/wiki/ Ant_colony_optimization.

Sorin C. Negulescu has a M.Sc. degree in Quality Mangement (2005) a M.Sc. degree in Computer Science (2003), "Lucian Blaga" University, Sibiu and a Ph. D in industrial engineering. His research interests include multi-agent systems with applications in manufacturing systems using stigmergic coordination, factory automation and the holonic paradigm.

Ioan Dzitac (b. February 14, 1953) received his M.Sc. in Mathematics (1977) and PhD in Information Sciences (2002) from "Babes-Bolyai" University of Cluj-Napoca. Now he is full professor of informatics at Mathematics-Informatics Department, Faculty of Exact Sciences, "Aurel Vlaicu" University of Arad, Romania and director of Research \& Development Agora Ltd. of Oradea, Romania. His current research interests include different aspects of Artificial Intelligence and Distributed Systems. He has (co-)authored 18 books and more than 70 papers, more than 50 de conferences participation, member in International Program Committee of more than 40 conferences and workshops.

Alina E. Lascu has a M.Sc. degree in International Relations and European Studies (2008), is currently in the last year of her master studies in the domain of Computer Science and in the first year of the doctoral studies in the domain of Computers and ICT. As depicted from her educational background her research interests are very much trans-disciplinary: Language Technology, Intelligent Agents, Agent Orientation, Computer-Aided Semiosis, Cultural and Interdisciplinary Studies. 